\title{
Relative Entropy of Random States and Black Holes
}

\author{
Jonah Kudler-Flam $\odot^{*}$ \\ Kadanoff Center for Theoretical Physics, University of Chicago, Chicago, Illinois 60637, USA
}

(Received 12 February 2021; accepted 2 April 2021; published 27 April 2021)

\begin{abstract}
We study the relative entropy of highly excited quantum states. First, we sample states from the Wishart ensemble and develop a large- $N$ diagrammatic technique for the relative entropy. The solution is exactly expressed in terms of elementary functions. We compare the analytic results to small- $N$ numerics, finding precise agreement. Furthermore, the random matrix theory results accurately match the behavior of chaotic many-body eigenstates, a manifestation of eigenstate thermalization. We apply this formalism to the AdS/CFT correspondence where the relative entropy measures the distinguishability between different black hole microstates. We find that black hole microstates are distinguishable even when the observer has arbitrarily small access to the quantum state, though the distinguishability is nonperturbatively small in Newton's constant. Finally, we interpret these results in the context of the subsystem eigenstate thermalization hypothesis (SETH), concluding that holographic systems obey SETH up to subsystems half the size of the total system.
\end{abstract}

DOI: 10.1103/PhysRevLett.126.171603

Introduction.-Random matrices are a unifying subject in quantum physics. From encoding quantum information [1], to characterizing complicated many-body systems and quantum chaos [2], to serving as toy models of the black hole information problem [3,4], random quantum states have become invaluable across many distinct subfields. Moreover, the mathematical field of random matrix theory is very mature, enabling analytical calculations in random states that would be otherwise intractable.

With the broad motivations of understanding structural properties of density matrices, quantum thermalization in isolated many-body systems, and the black hole information problem, we study the relative entropy of random quantum states. The applicability of this study to elucidating our motivating principles will subsequently be made clear.

The relative entropy between two density matrices $\rho$ and $\sigma$ is defined as

$$
D(\rho \| \sigma):=\operatorname{Tr}[\rho(\log \rho-\log \sigma)] .
$$

As a distinguishability measure, it obeys various nice properties, such as positivity with $D(\rho \| \sigma)=0$ if and only if $\rho=\sigma$. Crucially, the relative entropy is monotonic under quantum operations [5]

$$
D[\mathcal{N}(\rho) \| \mathcal{N}(\sigma)] \leq D(\rho \| \sigma),
$$

Published by the American Physical Society under the terms of the Creative Commons Attribution 4.0 International license. Further distribution of this work must maintain attribution to the author(s) and the published article's title, journal citation, and DOI. Funded by SCOAP ${ }^{3}$. where $\mathcal{N}$ is any completely positive trace-preserving map. A particularly important quantum operation that we will come back to is the partial trace. Monotonicity in this context means that density matrices become less distinguishable as you throw out more information about them, an intuitive notion.

Relative entropy is truly the mother of all quantities in quantum information theory. While at face value, it just measures the distinguishability between two density matrices, upon further inspection, its fundamental properties underlie many of the deepest universal statements about quantum mechanics [6,7], quantum field theory [8-10], and quantum gravity $[11,12]$.

While this progress has been significant, we will show that relative entropy has quite a bit more to tell us about each of these subdisciplines. (1) By finding a closed form solution for the relative entropy of random density matrices, we characterize the space of quantum states. In the language of quantum hypothesis testing $[13,14]$, this precisely determines the error that one achieves for a measure one set of quantum states when performing a hypothesis test with limited access to the quantum state. (2) While our general formula is exact in the limit of large Hilbert space dimensions, we find it to be remarkably accurate even for small Hilbert space dimensions. More interestingly, we find it to accurately predict the behavior of relative entropy between eigenstates of chaotic many-body Hamiltonians. These numerical observations imply that our results may be observable in noisy intermediate-scale quantum (NISQ) technologies [15]. (3) Through applying our formalism to holographic quantum field theories, we conclude that the relative entropy between subregions of black hole microstates is finite, though nonperturbatively 
small in Newton's constant $\left(G_{N}\right)$ up until the subregion is half of the total system size. Using quantum information inequalities, we show that this implies an extremely strong version of the eigenstate thermalization hypothesis [16,17].

Random mixed states. - We begin with a Haar random pure state on a bipartite Hilbert space $\mathcal{H}_{A} \otimes \mathcal{H}_{B}$ [18]

$$
|\Psi\rangle=\sum_{i=1}^{d_{A}} \sum_{\alpha=1}^{d_{B}} X_{i \alpha}|i\rangle_{A} \otimes|\alpha\rangle_{B},
$$

where the states in the sum are orthonormal bases for the sub-Hilbert spaces of dimensions $d_{A}$ and $d_{B}$ which we will always assume to be independently large. The $X_{i \alpha}$ 's are independently distributed complex Gaussian random variables with joint probability distribution $[18,19]$

$$
P\left(\left\{X_{i \alpha}\right\}\right)=\mathcal{Z}^{-1} \exp \left[-d_{A} d_{B} \operatorname{Tr}\left(X X^{\dagger}\right)\right],
$$

where $\mathcal{Z}$ is the normalization constant, ensuring the expression defines a probability. Here, $X$ represents the rectangular matrix whose matrix elements in the $i, \alpha$ basis are $X_{i \alpha}$. The random induced states on $\mathcal{H}_{A}$ are then

$$
\rho_{A}=\frac{X X^{\dagger}}{\operatorname{Tr}\left(X X^{\dagger}\right)} .
$$

We note that the denominator is a random variable that is sharply peaked around unity, so we can ignore it in the limit of large Hilbert space dimension $[18,19]$

$$
\rho_{A} \simeq X X^{\dagger} .
$$

This defines the Wishart ensemble. We now introduce a diagrammatic representation of the density matrix [20-22]

$$
[|\Psi\rangle\langle\Psi|]_{i \alpha, j \beta}=X_{i \alpha} X_{j \beta}^{*}=\begin{array}{cc}
i \alpha & \beta j \\
\mid & 1 \\
1 & 1
\end{array} \mid .
$$

The solid and dashed lines correspond to subsystems $A$ and $B$, respectively. Matrix manipulations are done at the bottom edge of the diagram. For example, the partial trace over $\mathcal{H}_{B}$ is

$$
\left[\rho_{A}\right]_{i, j}=\sum_{\alpha=1}^{d_{B}} X_{i \alpha} X_{j \alpha}^{*}:=\begin{array}{cr}
i \alpha & { }^{j} j \\
\left|'--^{\prime}\right|
\end{array} .
$$

Ensemble averaging is done at the top of the diagram with propagators carrying weight

$$
\text { , }:=\left\langle X_{i \alpha} X_{j \beta}^{*}\right\rangle=\frac{1}{d_{A} d_{B}} \delta_{i j} \delta_{\alpha \beta} .
$$

Putting these operations together, we can, for example, take the trace of the density matrix

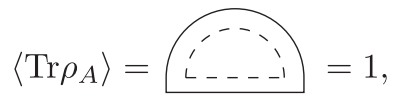

where every closed loop gives a factor of the Hilbert space dimension. The diagrammatic rules for averaging assert that we must sum over all possible contractions of the bras and kets. For relative entropy, we need two independent density matrices, $\rho_{A}$ and $\sigma_{A}$. These must be averaged over the ensemble separately. To make this distinction, we color $\sigma_{A}$ red.

The logarithms in the definition of relative entropy make the quantity significantly more difficult to compute analytically than simple powers of the density matrices. Happily, a replica trick for the relative entropy has been developed that reexpresses the logarithm as a limit of appropriate powers [23]

$$
D(\rho \| \sigma)=\lim _{n \rightarrow 1} \frac{1}{n-1}\left(\log \operatorname{Tr} \rho^{n}-\log \operatorname{Tr} \rho \sigma^{n-1}\right) .
$$

We will compute these two terms separately. The first term is recognized as minus the Rényi entropy. For $n=2$, we have

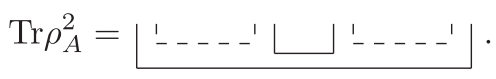

The ensemble average is a sum of the two contractions

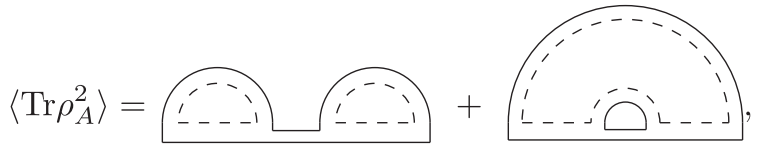

immediately giving $d_{A}^{-1}+d_{B}^{-1}$. This can be generalized to arbitrary powers. Because of the sum over all possible contractions, in general, the moments are expressible as a sum over the permutation group

$$
\left\langle\operatorname{Tr} \rho_{A}^{n}\right\rangle=\frac{1}{\left(d_{A} d_{B}\right)^{n}} \sum_{\tau \in S_{n}} d_{A}^{D\left(\eta^{-1} \circ \tau\right)} d_{B}^{D(\tau)},
$$

where $D(\cdots)$ is the number of cycles in the permutation and $\eta$ is the cyclic permutation. Each permutation corresponds to a diagram with the cycle structure determining which bra is contracted with which ket. For example, in Eq. (13), the first diagram corresponds to the identity permutation because each bra is contracted with its own ket while the second diagram corresponds to the swap permutation because the bra of the first density matrix is contracted with the ket of the second and vice versa. These are the only elements of $S_{2}$.

When the Hilbert spaces are large, only the terms that maximize $D\left(\eta^{-1} \circ \tau\right)+D(\tau)$ will contribute to the sum at leading order. These are known as the noncrossing permutations and have $D\left(\eta^{-1} \circ \tau\right)+D(\tau)=n+1$. Much is 
known about this special subset of permutations including that the number of such permutations with $D\left(\eta^{-1} \circ \tau\right)=k$ is given by the Narayana number $[24,25]$

$$
N_{n, k}=\frac{1}{n}\left(\begin{array}{l}
n \\
k
\end{array}\right)\left(\begin{array}{c}
n \\
k-1
\end{array}\right) \text {. }
$$

Thus, the sum can be reorganized as

$$
\begin{aligned}
\left\langle\operatorname{Tr} \rho_{A}^{n}\right\rangle & =\frac{1}{\left(d_{A} d_{B}\right)^{n}} \sum_{k=1}^{n} N_{n, k} d_{A}^{k} d_{B}^{n+1-k} \\
& =d_{A}^{1-n}{ }_{2} F_{1}\left(1-n,-n ; 2 ; \frac{d_{A}}{d_{B}}\right),
\end{aligned}
$$

where ${ }_{2} F_{1}$ is a hypergeometric function. This reproduces Page's famous result [26].

Next, we consider the second term of Eq. (11). For simplicity, we first consider the overlap between the two density matrices which, as a diagram, looks like

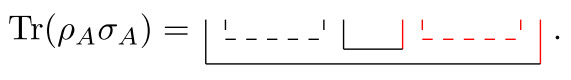

We must ensemble average the black and red lines separately, so there is only a single term

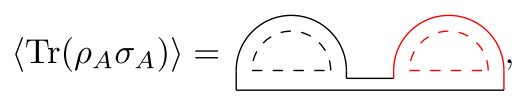

giving $d_{A}^{-1}$. We again generalize this to arbitrary powers by expressing the moments in terms of a sum over the permutation group

$$
\left\langle\operatorname{Tr}\left(\rho_{A} \sigma_{A}^{n-1}\right)\right\rangle=\frac{1}{\left(d_{A} d_{B}\right)^{n}} \sum_{\tau \in \mathbb{1} \times S_{n-1}} d_{A}^{D\left(\eta^{-1} \circ \tau\right)} d_{B}^{D(\tau)} .
$$

The crucial difference between this expression and Eq. (14) is that the sum is only over a subgroup of permutations, namely the ones that stabilize the first element. The first element (black lines) must be stabilized because the black lines must be contracted with themselves and there is only a single element with black lines. The reason the swap permutation was not included in Eq. (18) is because it acts nontrivially on the first density matrix.

The number of noncrossing permutations stabilizing the first element is given by the Narayana number, $N_{n-1, k}$. This can be seen from the diagrams which are topological in nature

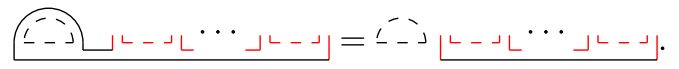

The diagrams maximizing the total number of loops are all of the noncrossing ones acting only on the $(n-1)$ red indices. We can then reorganize the sum as

$$
\left\langle\operatorname{Tr} \rho_{A} \sigma_{A}^{n-1}\right\rangle=\frac{1}{\left(d_{A} d_{B}\right)^{n}} \sum_{k=1}^{n-1} N_{n-1, k} d_{A}^{k} d_{B}^{n+1-k} .
$$

Like the Rényi entropies, this may also be written as a hypergeometric function,

$$
\left\langle\operatorname{Tr} \rho_{A} \sigma_{A}^{n-1}\right\rangle=d_{A}^{1-n}{ }_{2} F_{1}\left(1-n, 2-n ; 2 ; \frac{d_{A}}{d_{B}}\right) .
$$

Combining Eqs. (16) and (22), we can unambiguously take the $n \rightarrow 1$ limit to find the relative entropy [27]

$D\left(\rho_{A} \| \sigma_{A}\right)=1+\frac{d_{A}}{2 d_{B}}+\left(\frac{d_{B}}{d_{A}}-1\right) \log \left(1-\frac{d_{A}}{d_{B}}\right)$.

This is our main result. This formula is zero when $d_{A} / d_{B} \rightarrow 0$. This is to be expected because density matrices become indistinguishable when most of the information is "traced away." The relative entropy monotonically increases with $d_{A} / d_{B}$, reaching a curious value of $3 / 2$ when $d_{A}=d_{B}$. This monotonic behavior is a restatement of the monotonicity of relative entropy under the partial trace. For $d_{A}>d_{B}$, the density matrices are rank deficient leading to the formula breaking down and the relative entropy is formally infinite. We plot this function in Fig. 1 and compare to numerics, finding very good agreement even for the relatively small Hilbert space dimensions that are accessible on a classical computer.

We briefly comment on the implications of Eq. (23) for quantum hypothesis testing, which represented a breakthrough in the operational meaning of quantum relative entropy $[13,14]$. Say you are given a quantum state that is either $\rho$ or $\sigma$ and you wish to determine which one you have using quantum measurements. Quantum Stein's Lemma states that the optimal asymptotic rate of error

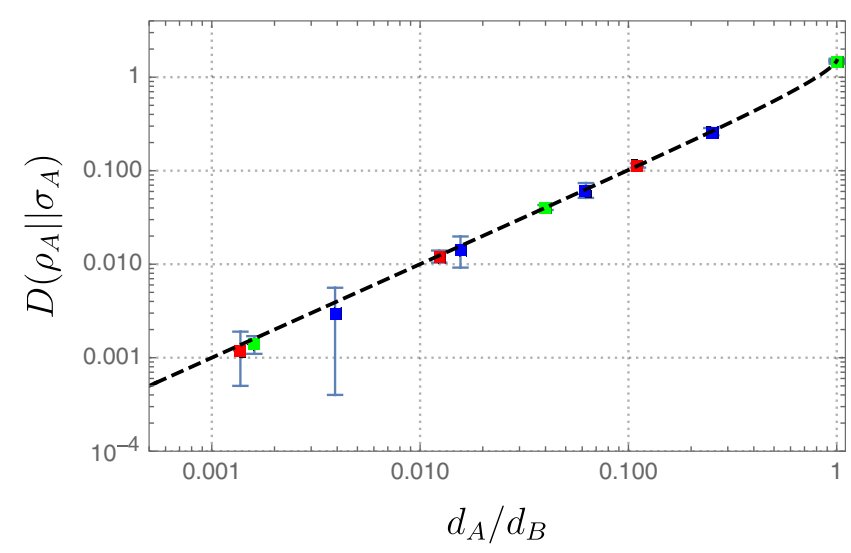

FIG. 1. Comparison of Eq. (23) (dashed line) with numerics. The blue, red, and green data points are for total Hilbert space dimensions of 1024, 6561, and 15625, respectively. The fluctuations in the relative entropy are clearly suppressed as the dimension is increased, signaling self-averaging. 
in determining which state you have is given by $e^{-D(\rho \| \sigma)}$ $[13,14]$. Thus, Eq. (23) tells us that if we are only given partial information about the state (access to sub-Hilbert space $A$ ), for a measure one set of quantum states, the error will either vanish if $A$ is larger than half the total system size or is finite and exponentially close (in the entropy) to the maximal error rate if $A$ is smaller than half the total system size.

Black hole microstates. - Here, we reinterpret Eq. (23) in the context of the AdS/CFT correspondence [31]. In AdS/CFT, high energy eigenstates in the boundary conformal field theory are dual to black hole microstates in the bulk because the correspondence is an isomorphism between the bulk and boundary Hilbert spaces. By black hole microstate, we therefore mean individual eigenstates of quantum gravity. Together, these microstates comprise the famous Bekenstein-Hawking entropy of the black hole $[32,33]$. Precise enumerations of these microstates have been performed for special black holes [34,35], though the general statement that the Bekenstein-Hawking entropy is truly a microscopic entropy is widely believed to be true.

Computations of relative entropy tell us how well we can distinguish different black hole microstates of similar energy, i.e., within the same microcanonical energy band [36], a notoriously difficult task that, a priori, one would expect to require knowledge of the full ultraviolet complete quantum gravity theory, such as string theory [34]. Surprisingly, we show that this is actually possible just from semiclassical gravity, which is related to the recent surprise that the Page curve can be calculated from semiclassical gravity $[39,40]$.

This is simplest for "fixed-area states" [41,42], which are holographic states where the areas of extremal surfaces in the bulk have been measured. These states have played an important role in understanding holographic entanglement entropy in the language of quantum error correction. While we first perform computations in the fixed-area state basis, we can translate these results to true energy eigenstates by noting that eigenstates are superpositions of fixed-area states with sharply peaked Gaussian distributions of width $O\left(\sqrt{G_{N}}\right)$ [43]. We will return to this translation in the discussion.

While many details can be found in the original papers and illuminating follow-ups [43-47], we will only present what is necessary for our analysis. We consider states where the areas of two extremal surfaces, $\gamma_{1}$ and $\gamma_{2}$, have been measured, as depicted in Fig. 2. The two surfaces wrap the black hole horizon in topologically distinct manners [48].

To compute the relative entropy between two different black hole microstates, we must compute

$$
\operatorname{Tr}\left(\rho_{A} \sigma_{A}^{n-1}\right)=\frac{\mathcal{Z}\left(\rho_{A} \sigma_{A}^{n-1}\right)}{\mathcal{Z}\left(\rho_{A}\right) \mathcal{Z}\left(\sigma_{A}\right)^{n-1}}
$$

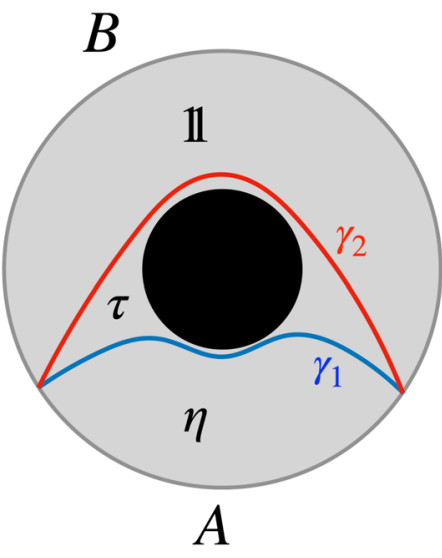

FIG. 2. Depicted is a black hole geometry with the boundary partitioned into regions $A$ and $B$. There are two competing extremal surfaces, $\gamma_{1}$ and $\gamma_{2}$, that we fix the area of. When performing the replica trick, we compute the path integral on $n$ copies of this geometry. Each bulk region is labeled by the permutation element that governs how it is glued among the copies.

where $\mathcal{Z}$ is the gravitational path integral with the boundary conditions dictated by the argument. Because of nice properties of fixed-area states, the only contributions to the path integrals come from the conical deficits that can occur at $\gamma_{1}$ and $\gamma_{2}$, leading to

$$
\operatorname{Tr}\left(\rho_{A} \sigma_{A}^{n-1}\right)=\sum_{\tau \in \mathbb{1} \times S_{n-1}} \frac{e^{\left(D\left(\eta^{-1} \circ \tau\right) A_{1}+D(\tau) A_{2}\right) / 4 G_{N}}}{e^{n\left(A_{1}+A_{2}\right) / 4 G_{N}}},
$$

where $A_{1}$ and $A_{2}$ are the areas of the fixed surfaces. This expression is identical to Eq. (19) once we identify $d_{A}=$ $e^{A_{1} / 4 G_{N}}$ and $d_{B}=e^{A_{2} / 4 G_{N}}$. A similar conclusion is made for $\operatorname{Tr}\left(\rho_{A}^{n}\right)$. Therefore, the relative entropy between black hole microstates is given by Eq. (23), which is UV finite because while the areas are themselves divergent, their difference is regulator independent. It is important to note that for small $A_{1}$, i.e., small boundary subregion $A$, the relative entropy is nonzero, meaning the two black hole microstates are distinguishable even with very limited information about the state. The catch is that the distinguishability is nonperturbatively small in Newton's constant, $O\left(e^{-1 / G_{N}}\right)$. However, as $A_{1}$ approaches $A_{2}$, the relative entropy becomes $O(1)$. The transition from $O\left(e^{-1 / G_{N}}\right)$ to $O(1)$ occurs in an extremely tiny window when $\left(A_{2}-A_{1}\right) / 4 G_{N} \lesssim \log 2$, roughly meaning that region $A$ contains one less qubit of information than region $B$.

In passing, we note that these results also apply to the relative entropy of two states in the Jackiw-Teitelboim gravity plus end-of-the-world brane model of black hole evaporation from Ref. [47] in the case that the black hole is in the microcanonical ensemble.

Subsystem eigenstate thermalization.-The subsystem eigenstate thermalization hypothesis $(\mathrm{ETH})$ is a 
generalization of the standard local ETH story and is significantly stronger. While the ETH is a statement about local operators $[16,17]$, the subsystem ETH is a statement that finite subregions appear thermal. Precisely, the subsystem ETH holds when sufficiently highly excited eigenstates have reduced density matrices that are exponentially close in trace distance to some universal density matrices, such as the microcanonical ensemble [51]

$$
T\left(\rho_{\psi}, \rho_{\text {univ }}\right):=\left|\rho_{\psi}-\rho_{\text {univ }}\right|_{1} \leq O\left(e^{-S(E) / 2}\right),
$$

where $e^{S(E)}$ is the density of states of the full system. In the context of holography, the entropy scales as $O\left(G_{N}^{-1}\right)$, so the subsystem ETH means that the trace distance is nonperturbatively small in Newton's constant.

To prove this, we now invoke the quantum Pinsker inequality [52]

$$
D(\rho \| \sigma) \geq \frac{1}{2} T(\rho, \sigma)^{2} .
$$

We previously found $D\left(\rho_{A} \| \sigma_{A}\right)$ to scale as $O\left(e^{-1 / G_{N}}\right)$ for any two black hole microstates with fixed area. This implies that the trace distance is, at most, $O\left(e^{-1 / G_{N}}\right)$. The trace distance defines a metric on the space of density matrices, so if a typical state is close to a measure one set of all other states, then the universal density matrix should sit within this ball. We therefore claim that fixed-area states in all dimensions obey the subsystem ETH for subsystems less than half the total system size. The violation of the subsystem ETH only occurs when $\left(A_{2}-A_{1}\right) / 4 G_{N} \lesssim \log 2$.

Discussion.-There are various interesting directions that one may take: (i) We have computed the average relative entropy between typical random mixed states. However, we have not fixed the complete distribution. It would be interesting to characterize the fluctuations in relative entropy. Higher moments of the relative entropy can be computed using the same technology that we have developed. (ii) In our applications to holography, we focused on fixed-area states. More generic states are superpositions of fixed-area states. It is important to study the relative entropy of these more generic states to verify that it is qualitatively similar. We can invoke the joint convexity of the trace distance [53]

$T\left(\sum_{i} p_{i} \rho_{i}, \sum_{i} q_{i} \sigma_{i}\right) \leq T\left(p_{i}, q_{i}\right)+\sum_{i} p_{i} T\left(\rho_{i}, \sigma_{i}\right)$,

where the $\rho_{i}$ and $\sigma_{i}$ 's are fixed-area states and $T\left(p_{i}, q_{i}\right)$ is the classical trace distance between probability distributions. We have already shown that the second term on the right-hand side is $O\left(e^{-1 / G_{N}}\right)$. If we assume that the probability distributions are Gaussian with equal widths but centered at fixed areas a distance at most $O\left(e^{-1 / G_{N}}\right)$ apart, i.e., within the same microcanonical window, then it is a straightforward exercise to confirm that the first term is also $O\left(e^{-1 / G_{N}}\right)$, confirming the subsystem ETH [54]. However, if the widths of the Gaussian distributions are different, even by an amount polynomial in $G_{N}$, the bound will no longer be tight. It would be fascinating if these corrections could lead to violations of eigenstate thermalization. (iii) One of our motivations to study random states is that they should be representative of generic excited states in chaotic quantum systems. It is clearly interesting to check how accurately our results characterize real Hamiltonian systems (beyond holography). We provide numerical results for the Sachdev-Ye-Kitaev (SYK) model and for spin chains in the supplemental material [28]. While the SYK eigenstates mimic random matrix theory, we find that chaotic spin chain eigenstates have close to, but larger, relative entropy than random states and integrable eigenstates have even larger relative entropy and much larger fluctuations. We hope to report a more systematic study in the future.

I am grateful to Chris Akers, Hong Liu, Pratik Rath, Shinsei Ryu, Hassan Shapourian, and Shreya Vardhan for helpful discussions and comments and to Kazumi Okuyama for explaining how to simplify the functional form of Eq. (23). I am supported through a Simons Investigator Award to Shinsei Ryu from the Simons Foundation (Grant No. 566166).

Note added.-Recently, I became aware of an independent project with similar results on the computation of relative entropy [55].

jkudlerflam@uchicago.edu

[1] B. Collins and I. Nechita, Random matrix techniques in quantum information theory, J. Math. Phys. (N.Y.) 57, 015215 (2016).

[2] L. D'Alessio, Y. Kafri, A. Polkovnikov, and M. Rigol, From quantum chaos and eigenstate thermalization to statistical mechanics and thermodynamics, Adv. Phys. 65, 239 (2016).

[3] D. N. Page, Information in Black Hole Radiation, Phys. Rev. Lett. 71, 3743 (1993).

[4] P. Hayden and J. Preskill, Black holes as mirrors: Quantum information in random subsystems, J. High Energy Phys. 09 (2007) 120.

[5] G. Lindblad, Completely positive maps and entropy inequalities, Commun. Math. Phys. 40, 147 (1975).

[6] G. Lindblad, Expectations and entropy inequalities for finite quantum systems, Commun. Math. Phys. 39, 111 (1974).

[7] V. Vedral, The role of relative entropy in quantum information theory, Rev. Mod. Phys. 74, 197 (2002).

[8] H. Casini and M. Huerta, A finite entanglement entropy and the c-theorem, Phys. Lett. B 600, 142 (2004).

[9] T. Faulkner, R. G. Leigh, O. Parrikar, and H. Wang, Modular Hamiltonians for deformed half-spaces and the averaged null energy condition, J. High Energy Phys. 09 (2016) 038. 
[10] S. Balakrishnan, T. Faulkner, Z. U. Khandker, and H. Wang, A general proof of the quantum null energy condition, J. High Energy Phys. 09 (2019) 020.

[11] H. Casini, Relative entropy and the Bekenstein bound, Classical Quantum Gravity 25, 205021 (2008).

[12] A. C. Wall, Proof of the generalized second law for rapidly changing fields and arbitrary horizon slices, Phys. Rev. D 85, 104049 (2012).

[13] F. Hiai and D. Petz, The proper formula for relative entropy and its asymptotics in quantum probability, Commun. Math. Phys. 143, 99 (1991).

[14] T. Ogawa and H. Nagaoka, Strong converse and Stein's lemma in quantum hypothesis testing, in Asymptotic Theory of Quantum Statistical Inference: Selected Papers, edited by H. Masahito (World Scientific Publishing Co. Pte. Ltd, Singapore, 2005), pp. 28-42.

[15] J. Preskill, Quantum computing in the NISQ era and beyond, Quantum 2, 79 (2018).

[16] J. M. Deutsch, Quantum statistical mechanics in a closed system, Phys. Rev. A 43, 2046 (1991).

[17] M. Srednicki, Chaos and quantum thermalization, Phys. Rev. E 50, 888 (1994).

[18] B. Collins and I. Nechita, Gaussianization and eigenvalue statistics for random quantum channels (III), Ann. Appl. Probab. 21, 1136 (2011).

[19] K. Zyczkowski and H.-J. Sommers, Induced measures in the space of mixed quantum states, J. Phys. A 34, 7111 (2001).

[20] E. Brézin and A. Zee, Universal relation between Green functions in random matrix theory, Nucl. Phys. B453, 531 (1995).

[21] J. Jurkiewicz, G. Łukaszewski, and M. A. Nowak, Diagrammatic approach to fluctuations in the Wishart ensemble, Acta Phys. Polon. B 39, 799 (2008).

[22] H. Shapourian, S. Liu, J. Kudler-Flam, and A. Vishwanath, Entanglement negativity spectrum of random mixed states: A diagrammatic approach, arXiv:2011.01277.

[23] N. Lashkari, Modular Hamiltonian for Excited States in Conformal Field Theory, Phys. Rev. Lett. 117, 041601 (2016).

[24] G. Kreweras, Sur les partitions non croisees d'un cycle, Discr. Math. 1, 333 (1972).

[25] R. Simion, Noncrossing partitions, Discr. Math. 217, 367 (2000).

[26] D. N. Page, Average Entropy of a Subsystem, Phys. Rev. Lett. 71, 1291 (1993).

[27] In general, the ensemble average and logarithm do not commute, requiring a further replica trick. However, these operations approximately commute for large Hilbert space dimensions. We show this explicitly in the supplemental material [28], which includes Refs. [25,26,29,30].

[28] See Supplemental Material at http://link.aps.org/ supplemental/10.1103/PhysRevLett.126.171603 for the details of computations and numerical results for Hamiltonian systems.

[29] M. C. Bañuls, J. I. Cirac, and M. B. Hastings, Strong and Weak Thermalization of Infinite Nonintegrable Quantum Systems, Phys. Rev. Lett. 106, 050405 (2011).

[30] A. Kitaev, A simple model of quantum holography, in KITP Program: Entanglement in Strongly-Correlated
Quantum Matter (2015), https://online.kitp.ucsb.edu/ online/entangled15/kitaev/.

[31] J. Maldacena, The large-N limit of superconformal field theories and supergravity, Int. J. Theor. Phys. 38, 1113 (1999).

[32] J. D. Bekenstein, Black holes and the second law, Lett. Nuovo Cim. 4, 737 (1972).

[33] S. W. Hawking, Particle creation by black holes, Commun. Math. Phys. 43, 199 (1975); Erratum, Commun. Math. Phys. 46, 206 (1976).

[34] A. Strominger and C. Vafa, Microscopic origin of the Bekenstein-Hawking entropy, Phys. Lett. B 379, 99 (1996).

[35] A. Strominger, Black hole entropy from near-horizon microstates, J. High Energy Phys. 02 (1998) 009.

[36] Related questions were studied in Refs. [37,38].

[37] N. Bao and H. Ooguri, Distinguishability of black hole microstates, Phys. Rev. D 96, 066017 (2017).

[38] G. Sárosi and T. Ugajin, Relative entropy of excited states in conformal field theories of arbitrary dimensions, J. High Energy Phys. 02 (2017) 060.

[39] G. Penington, Entanglement wedge reconstruction and the information paradox, J. High Energy Phys. 09 (2020) 002 .

[40] A. Almheiri, N. Engelhardt, D. Marolf, and H. Maxfield, The entropy of bulk quantum fields and the entanglement wedge of an evaporating black hole, J. High Energy Phys. 12 (2019) 063.

[41] C. Akers and P. Rath, Holographic Renyi entropy from quantum error correction, J. High Energy Phys. 05 (2019) 052.

[42] X. Dong, D. Harlow, and D. Marolf, Flat entanglement spectra in fixed-area states of quantum gravity, J. High Energy Phys. 10 (2019) 240.

[43] D. Marolf, S. Wang, and Z. Wang, Probing phase transitions of holographic entanglement entropy with fixed area states, J. High Energy Phys. 12 (2020) 084.

[44] X. Dong and D. Marolf, One-loop universality of holographic codes, J. High Energy Phys. 03 (2020) 191.

[45] X. Dong and H. Wang, Enhanced corrections near holographic entanglement transitions: A chaotic case study, J. High Energy Phys. 11 (2020) 007.

[46] C. Akers and G. Penington, Leading order corrections to the quantum extremal surface prescription, arXiv:2008.03319.

[47] G. Penington, S. H. Shenker, D. Stanford, and Z. Yang, Replica wormholes and the black hole interior, arXiv: 1911.11977.

[48] In fact, the conclusions from this section hold whenever there exist more than one extremal surface in the bulk geometry (see, e.g., Ref. [46]). The black hole is not strictly necessary though we view it as the most physically relevant application. The existence of multiple extremal surfaces in the black hole geometry can be seen as a consequence of Ref. [49]. See Ref. [50] for explicit constructions.

[49] N. Engelhardt and A. C. Wall, Extremal surface barriers, J. High Energy Phys. 03 (2014) 068.

[50] V. E. Hubeny, H. Maxfield, M. Rangamani, and E. Tonni, Holographic entanglement plateaux, J. High Energy Phys. 08 (2013) 092.

[51] A. Dymarsky, N. Lashkari, and H. Liu, Subsystem eigenstate thermalization hypothesis, Phys. Rev. E 97, 012140 (2018). 
[52] M. Ohya and D. Petz, Quantum Entropy and Its Use, Theoretical and Mathematical Physics (Springer Berlin Heidelberg, Berlin Heidelberg, 2004).

[53] M. A. Nielsen and I. L. Chuang, Quantum Computation and Quantum Information: 10th Anniversary Edition (Cambridge University Press, Cambridge, England, 2010).
[54] The positivity of the trace distance immediately follows from assuming the Gaussian means to be different because $T(\rho, \sigma)=0$ only if $\rho=\sigma$.

[55] R. Caginalp, M. Moosa, and P. Rath (private communication). 